Pacific Journal of Mathematics

QUOTIENTS BY COMPLEX CONJUGATION OF
NONSINGULAR QUADRICS AND CUBITS IN $P_{C}^{3}$ DEFINED

Maurizio Letizia 


\title{
QUOTIENTS BY COMPLEX CONJUGATION OF NONSINGULAR QUADRICS AND CUBICS IN $\mathbf{P}_{\mathbf{C}}^{3}$ DEFINED OVER $\mathbf{R}$
}

\author{
MaUrizio Letizia
}

\begin{abstract}
If $X$ is a complex algebraic variety defined over $R$, complex conjugation in $C$ induces an involution $\tau: X \rightarrow X$ which we shall still call complex conjugation. If $X$ is nonsingular and of complex dimension $2, \tau$ is an orientation preserving diffeomorphism and the quotient $X / \tau$ of $X$ by $\tau$ is, as $X$, a naturally oriented smooth manifold without boundary. Our aim is to describe $X / \tau$, up to diffeomorphisms, in case $X$ is a nonsingular quadric or cubic in $\mathbf{P}_{\mathbf{C}}^{3}$.
\end{abstract}

Our results can be summarized in the following:

Proposition. If $X$ is a nonsingular quadric or cubic in $\mathbf{P}_{\mathbf{C}}^{3}$ defined over $\mathbf{R}$ then $X / \tau$ is, up to diffeomorphisms, obtained from the 4-sphere $S^{4}$ by a connected sum with copies of $\overline{\mathbf{P}}_{\mathrm{C}}^{2}$.

The proof is based on an analysis of the change of diffeomorphism type of $X / \tau$ when $X$ varies in a generic pencil of surfaces of degree $d$ of $\mathbf{P}_{\mathbf{C}}^{3}$ defined over $\mathbf{R}$ (see §2).

Our results can be seen as an extension of Kuiper's theorem [3], which says that $\mathbf{P}_{\mathbf{C}}^{2} / \tau$ is diffeomorphic to $S^{4}$, in the direction of a discussion of the general problem of describing the quotients by conjugation of nonsingular surfaces of $\mathbf{P}_{\mathbf{C}}^{3}$ defined over $\mathbf{R}$.

A propos we remind the reader that the diffeomorphism type of the real loci of such surfaces has been classified only when their degree is $\leq 4$ (actually in the degree 4 case there are still some lacunae [2]) and practically nothing is known for higher degrees. Nevertheless we feel that some general statements about the diffeomorphisms type of $X / \tau$ might possibly be proved independently of such classification. For instance it might turn out to be possible to single out in each degree $d$ a reasonably small set of manifolds such that every $X / \tau$ is obtained from one of them by a connected sum with copies of $\overline{\mathbf{P}}_{\mathbf{C}}^{2}$. By the way we mention that in degree 4 it seems that the set consisting of $S^{2} \times S^{2}$ and $\mathbf{P}_{\mathbf{C}}^{2}$ has this property.

Anyhow before starting the proof of the proposition we want to recall some general facts which place the particular cases we shall be dealing with into perspective at least from a purely topological point of view. 
If $X$ is any complex algebraic variety defined over $\mathbf{R}$ the natural projection $\pi: X \rightarrow X / \tau$ is a 2-covering branched along the real locus $X_{\mathrm{R}}$ of $X$. If $X$ is smooth connected with $\operatorname{dim} X_{\mathbf{C}} \geq 2$ and $X_{\mathrm{R}} \neq \varnothing, \pi_{*}: \pi_{1}(X) \rightarrow$ $\pi_{1}(X / \tau)$ is surjective. The cohomology ring $H^{*}(X / \tau, F)$, with coefficients in any field $F$ of characteristic $\neq 2$, is mapped isomorphically by $\pi^{*}$ onto the subring of $H^{*}(X, F)$ consisting of the elements left fixed by $\tau$. Moreover, one has, for each $n \geq 0$, estimates

(*) $\operatorname{dim} H^{n}\left(X / \tau, \pi\left(X_{\mathbf{R}}\right), \mathbf{Z}_{2}\right)+\sum_{i \geq n} \operatorname{dim} H^{i}\left(X_{\mathbf{R}}, \mathbf{Z}_{2}\right) \leq \sum_{i \geq n} H^{i}\left(X \mathbf{Z}_{2}\right)$

provided by Smith theory ([1]). Whatever the characteristic of $F$, we have the following relationship for the Euler-Poincare characteristics:

$$
\chi\left(X_{\mathbf{R}}, F\right)=2 \chi(X / \tau, F)-\chi(X, F) .
$$

If $X$ is projective nonsingular, $\tau^{*}$ maps $C^{\infty}$ forms of type $(p, q)$ into ones of type $(q, p)$, and, in case $\operatorname{dim}_{\mathbf{C}} X=2, \pi^{*}$ maps the cohomology class of a hyperplane section defined over $\mathbf{R}$ into minus itself. Hence we have, in this case, $\operatorname{dim}_{\mathbf{R}} H^{i}(X / \tau, \mathbf{R})=\frac{1}{2} \operatorname{dim} H^{i}(X, \mathbf{R})$ for $i=1$ or 3 . Let us write

$$
H^{2}(X / \tau, \mathbf{R})=H^{2}(X / \tau, \mathbf{R})^{+} \oplus H^{2}(X / \tau, \mathbf{R})^{-},
$$

the sum being orthogonal with respect to the intersection form, this one being positive definite on the first addend and negative definite on the second. Since the intersection pairing of $H^{2}(X, \mathbf{R})$ is positive definite on $H^{2}(X, \mathbf{R}) \cap\left(H^{0}\left(X, \Omega_{x}^{2}\right) \oplus H^{2}\left(X, O_{x}\right)\right)$ and negative definite on the orthogonal complement of the hyperplane class in $H^{2}(X, \mathbf{R}) \cap H^{1}\left(X, \Omega_{x}^{1}\right)$, we have:

$$
\begin{array}{r}
\operatorname{dim}_{\mathbf{R}} H^{2}(X / \tau, \mathbf{R})^{+}=\operatorname{dim}_{\mathbf{C}} H^{0}\left(X, \Omega_{X}^{2}\right) \\
\quad\left(=\left(\begin{array}{c}
d-1 \\
3
\end{array}\right) \text { if } X \subseteq \mathbf{P}_{\mathbf{C}}^{3} \text { of degree } d\right) ; \\
\operatorname{dim}_{\mathbf{R}} H^{2}(X / \tau, \mathbf{R})^{-} \leq \operatorname{dim}_{\mathbf{C}} H^{1}\left(X, \Omega_{X}^{1}\right)-1 \\
\left(=\frac{1}{3}\left(2 d^{3}-6 d^{2}+7 d\right)-1 \text { if } X \subseteq \mathbf{P}_{\mathbf{C}}^{3} \text { of degree } d\right) .
\end{array}
$$

Here $\Omega_{X}^{i}$ denotes the sheaf of holomorphic $i$-forms on $X$. In particular if $X$ is a quadric or a cubic in $\mathbf{P}_{\mathbf{C}}^{3}$ the intersection form is negative definite on $H^{2}(X / \tau, \mathbf{R})$. Adding $(* *)$ and $(*)$, for $n=0$, we get an estimate for the number of connected components of $X_{\mathbf{R}}$ for a $X \subseteq \mathbf{P}_{\mathbf{C}}^{3}$ of degree $d$, namely

$$
\operatorname{dim}_{\mathbf{z}_{2}} H^{0}\left(X_{\mathbf{R}} \mathbf{Z}_{2}\right) \leq \frac{1}{2}\left[d^{3}-4 d^{2}+6 d-1-\left(\begin{array}{c}
d-1 \\
3
\end{array}\right)\right]
$$


(compare [4]). On the other hand if we suppose $X_{\mathbf{R}}$ connected we must have

$$
\operatorname{dim}_{\mathbf{R}} H^{1}\left(X_{\mathbf{R}}, \mathbf{R}\right) \leq \frac{1}{3}\left(2 d^{3}-6 d^{2}+7 d\right)
$$

(actually we can add a -1 to the right side if $d$ is odd; notice that this estimate is in fact sharp if $d=2,3$ ).

Let us incidentally observe that if $\operatorname{dim}_{\mathbf{C}} X=1$ and $X$ is of genus $g$, by a previous remark we must have $\operatorname{dim}_{\mathbf{R}} H^{1}(X / \tau, \mathbf{R})=g$. Now the components of $X_{\mathbf{R}}$ become boundary components of $X / \tau$, and for any compact smooth real surface $S$ with boundary, $\operatorname{dim}_{\mathbf{R}} H^{1}(S, \mathbf{R})+1$ is an upper bound for the number of its boundary components so we get Harnack's theorem.

2. Let us parametrize as usual the set of all surfaces of degree $d$ in $\mathbf{P}_{\mathbf{C}}^{3}$ defined over $\mathbf{R}$ by points of

$$
\mathbf{P}_{\mathbf{R}}^{\left(\frac{d+3}{3}\right)-1}
$$

and let

$$
\Delta^{(d)} \subseteq \mathbf{P}_{\mathbf{C}}^{(d+3)-1}
$$

be the discriminant hypersurface of the surfaces of degree $d$. If $X_{1}$ and $X_{2}$ are parametrized by points belonging to the same topological connected component of

$$
\mathbf{P}_{\mathbf{R}}^{(d+3)-1}-\Delta_{\mathbf{R}}^{(d)}
$$

there is a diffeomorphism $\phi: X_{1} \rightarrow X_{2}$ which commutes with $\tau$ so that $X_{1} / \tau$ and $X_{2} / \tau$ are diffeomorphic. We now want to investigate what relationship there is between $X_{1} / \tau$ and $X_{2} / \tau$ when $X_{1}$ and $X_{2}$ belong to two different but adjacent components of

$$
\mathbf{P}_{\mathbf{R}}^{\left(\frac{d+3}{3}\right)-1}-\Delta_{\mathbf{R}}^{(d)}
$$

We can assume there is a family $\left\{X_{\lambda}\right\}, \lambda \in \mathbf{R},|\lambda|<\varepsilon$, of surfaces given in some affine system of coordinates by $f(x y z)-\lambda=0$ such that $X_{\lambda}$ is nonsingular for $\lambda \neq 0 ; X_{0}$ has a unique singular point which is at $(0,0,0)$ and is a nondegenerate quadratic point; and $X_{1}$ is $X_{\lambda_{1}}$ for any $\lambda_{1}<0$ and $X_{2}$ is $X_{\lambda_{2}}$ for any $\lambda_{2}>0$.

Furthermore, if $B_{\delta}=\left\{\left.(x, y, z) \in \mathbf{C}^{3}|| x\right|^{2}+|y|^{2}+|z|^{2}<\delta\right\}$, we can assume $\varepsilon$ and $\delta$ so chosen that there is a diffeomorphism between $X_{1}-B_{\delta} \cap X_{1}$ and $X_{2}-B_{\delta} \cap X_{2}$ commuting with $\tau$ and there is a diffeomorphism commuting with $\tau$ of $\bar{B}_{\delta}$ onto itself carrying $X_{\lambda} \cap \bar{B}_{\delta}$ onto 
$Y_{\lambda} \cap \bar{B}_{\delta}$, the family $\left\{Y_{\lambda}\right\}, \lambda \in \mathbf{R},|\lambda|<\varepsilon$, being given either by $x^{2}+y^{2}+$ $z^{2}=\lambda$ (we shall refer to this as the first case) or by $x^{2}+y^{2}-z^{2}=\lambda$ (second case). (Since $f$ restricted to $\mathbf{R}^{3}$ is real analytic, the Morse lemma provides a real analytic change of coordinates in a neighborhood of $(0,0,0)$ in $\mathbf{R}^{3}$ which reduces $f$ to the quadratic part of its Taylor expansion: this change of coordinates can be extended to a complex analytic change of coordinates in a neighborhood of $(0,0,0)$ in $\mathbf{C}^{3}$ which will serve our purposes.)

Let us write $x=x_{1}+i x_{2}, y=y_{1}+i y_{2}, z=z_{1}+i z_{2}$ with $x_{i}, y_{l}, z_{l} \in$ R. In the first case, and if $\lambda>0, x^{2}+y^{2}+z^{2}=\lambda$ is equivalent to the system:

$$
x_{1}^{2}+y_{1}^{2}+z_{1}^{2}=\lambda+x_{2}^{2}+y_{2}^{2}+z_{2}^{2}, \quad x_{1} x_{2}+y_{1} y_{2}+z_{1} z_{2}=0,
$$

and the map

$$
\left(x_{1}, x_{2}, y_{1}, y_{2}, z_{1}, z_{2}\right) \rightarrow\left(\frac{x_{1}}{D}, \frac{y_{1}}{D}, \frac{z_{1}}{D}, x_{2}, y_{2}, z_{2}\right),
$$

where $D=\sqrt{\lambda+x^{2}+y^{2}+z^{2}}$, exhibits $Y_{\lambda} \cap B_{\delta}$, if $\lambda$ is small enough, as diffeomorphic to the tangent bundle $\Upsilon S^{2}$ of a 2-sphere $S^{2}$; through this diffeomorphism complex conjugation becomes the involution of $\Upsilon S^{2}$, which is the identity on the base and multiplication by -1 on the fibres. Considering $S^{2}$ as $\mathbf{P}_{\mathbf{C}}^{1}, \Upsilon S^{2}$ as the bundle associated to the sheaf $O_{\mathbf{P}_{\mathbf{C}}^{1}}(2)$ and the map $O_{\mathbf{P}_{\mathbf{C}}^{\mathbf{l}}}(2) \rightarrow O_{\mathbf{P}_{\mathbf{C}}^{\mathbf{l}}}(4)$ given by $\sigma \rightarrow \sigma^{2}$, we see that $Y_{\lambda} \cap B_{\delta} / \tau$ is diffeomorphic to the degree 4 complex line bundle over $\mathbf{P}_{\mathbf{C}}^{1}$. If $\lambda<0$ we have again, in an analogous way, a diffeomorphism of $Y_{\lambda} \cap B_{\delta}$ with $\Upsilon S^{2}$; this time, however, conjugation becomes the antipodal map on the basis and minus the map induced on $\Upsilon S^{2}$ by the antipodal map on the fibres so that $Y_{\lambda} \cap B_{\delta} / \tau$ is diffeomorphic to the degree 1 disk bundle over $\mathbf{P}_{\mathbf{R}}^{2}$, i.e., to the normal bundle to $\mathbf{P}_{\mathbf{R}}^{2}$ in $\mathbf{P}_{\mathbf{C}}^{2}$.

In the second case we also have a diffeomorphism of $Y_{\lambda} \cap B_{\delta}$ with $\Upsilon S^{2}$, but complex conjugation now becomes reflection around an equatorial plane on the base and minus the map induced by this reflection on the fibres if $\lambda>0$, and a rotation of angle $\pi$ on the base and minus the map induced by such rotation on the fibres if $\lambda<0$, so that $Y_{\lambda} \cap B_{\delta} / \tau$ is diffeomorphic to a 4-ball $B^{4}$ if $\lambda>0$, and to the degree 1 complex line bundle over $\mathbf{P}_{\mathbf{C}}^{1}$ if $\lambda<0$. If we also keep track of the orientations we see therefore that in the second case there is an orientation preserving diffeomorphism between $X_{2} / \tau$ and $X_{1} / \tau \# \overline{\mathbf{P}}_{2}^{2}$ - where as usual \# stands for the operation of connected sum and $\overline{\mathbf{P}}_{\mathbf{C}}^{2}$ for $\mathbf{P}_{\mathbf{C}}^{2}$ endowed with the orientation opposite to the standard one. In the first case we can only say that $X_{2} / \tau$ is obtained from $X_{1} / \tau$ by removing a degree 1 disk bundle over $\mathbf{P}_{\mathbf{R}}^{2}$ and putting in its place a degree 4 complex line bundle over $\mathbf{P}_{\mathbf{C}}^{1}$. 
3. Let us now deal with the first specific objective of this paper: there are 3 connected families of nonsingular quadrics in $\mathbf{P}_{\mathbf{C}}^{3}$ defined over $\mathbf{R}$, a representative of each, say $Q_{1}, Q_{2}, Q_{3}$, being given by the equation $x_{3}^{2}+x_{2}^{2}+x_{1}^{2}+x_{0}^{2}=0, x_{3}^{2}+x_{2}^{2}+x_{1}^{2}-x_{0}^{2}=0, x_{3}^{2}+x_{2}^{2}-x_{1}^{2}-x_{0}^{2}=$ 0 , respectively. It is $Q_{1 \mathbf{R}}=\varnothing, Q_{2 \mathbf{R}} \approx S^{2}, Q_{3 \mathbf{R}} \approx \mathbf{P}_{\mathbf{R}}^{1} \times \mathbf{P}_{\mathbf{R}}^{1}$, where $\approx$ stands for "is diffeomorphic to".

There is an algebraic isomorphism defined over $\mathbf{R}$ between $Q_{3}$ and $\mathbf{P}_{\mathbf{C}}^{1} \times \mathbf{P}_{\mathbf{C}}^{1}$. If we let complex conjugation act separately on the factors of $\mathbf{P}_{\mathbf{C}}^{1} \times \mathbf{P}_{\mathbf{C}}^{1}$ we get a group of order $4, G$, of diffeomorphisms of this manifold and we have:

$$
\mathbf{P}_{\mathbf{C}}^{1} \times \mathbf{P}_{\mathbf{C}}^{1} / G \approx \mathbf{P}_{\mathbf{C}}^{1} / \tau \times \mathbf{P}_{\mathbf{C}}^{1} / \tau \approx \bar{B}^{2} \times \bar{B}^{2} \approx \bar{B}^{4}
$$

( $\bar{B}^{i}$ is the closed $i$-ball).

Hence we obtain a map $\mathbf{P}^{1} \times \mathbf{P}^{1} / \tau \rightarrow \bar{B}^{4}$ which exhibits $\mathbf{P}^{1} \times \mathbf{P}^{1} / \tau$ as a double cover of $\bar{B}^{4}$ branched precisely along the boundary of $\bar{B}^{4}$ : we are so led to the conclusion that $Q_{3} / \tau$ is diffeomorphic to the 4-sphere $S^{4}$.

Considering the family $x_{3}^{2}+x_{2}^{2}+\lambda x_{1}^{2}-x_{0}^{2}=0$ we get from $\S 2$ that $Q_{2} / \tau$ is diffeomorphic to $S^{4} \# \overline{\mathbf{P}}_{\mathbf{C}}^{2}$, i.e. to $\overline{\mathbf{P}}_{\mathbf{C}}^{2}$. Again consideration of the family $x_{3}^{2}+x_{2}^{2}+x_{1}^{2}+\lambda x_{0}^{2}=0$ shows that $Q_{1} / \tau$ is obtained from $\overline{\mathbf{P}}_{\mathbf{C}}^{2}$ by removing a certain degree 4 complex line bundle over $\mathbf{P}_{\mathbf{C}}^{1}$ and replacing it with a degree 1 disk bundle over $\mathbf{P}_{\mathbf{R}}^{2}$; actually we can describe the complement of that degree 4 complex line bundle over $\mathbf{P}_{\mathbf{C}}^{1}$. In fact $X_{\lambda}-X_{\lambda} \cap B_{\delta}$ is diffeomorphic to the normal bundle of the hyperplane section at infinity which is, in this case, again diffeomorphic to $\Upsilon S^{2}$ through a diffeomorphism which carries complex conjugation into the map which is the antipodal map on the base and minus the map induced by the antipodal map on the fibres. This more or less already follows from the fact that the degree of the normal bundle of the hyperplane section at infinity equals its self-intersection, which in our case is 2 , and the fact that the hyperplane section at infinity has no real points. Anyhow we can exhibit an explicit diffeomorphism with the stated properties between $V_{0}-\{(1: 0: 0: 0)\}$ and $\Upsilon S^{2}$ as follows: write $X_{i}=X_{i}^{1}+i X_{i}^{2}$ with $X_{i}^{j} \in \mathbf{R}$ and consider $X_{i}=\left(x_{1}^{i}, x_{2}^{i}, x_{3}^{i}\right)(i=1,2)$ as vectors of $\mathbf{R}^{3}$. The map $\phi$ : $V_{0}-\{(1: 0: 0: 0)\} \rightarrow \mathbf{R}^{3} \times \mathbf{R}^{3}$ given by

$$
\phi\left(\left(x_{0}: x_{1}: x_{2}: x_{3}\right)\right)=\left(\frac{X_{1} \wedge X_{2}}{\left\|X_{1} \wedge X_{2}\right\|}, \frac{x_{3}^{1} X_{1}+x_{3}^{2} X_{2}}{\left\|X_{1} \wedge X_{2}\right\|}\right),
$$

where $\wedge$ stands for the ordinary vector product and \| $\|$ for the ordinary norm, has the desired properties. In conclusion we have that the complement in question is the degree 1 disk bundle over $\mathbf{P}_{\mathbf{R}}^{2}$ and $Q_{1} / \tau$ is the double of this bundle. 
Before passing on to deal with cubics we want to remark that it is possible to give another proof of Kuiper's theorem ([2]) in the following way: if $\varepsilon: \hat{Q}_{3} \rightarrow Q_{3}$ is the blowing up of $Q_{3}$ in one of its real points $P$, projection of $Q_{3}$ from $P$ onto a complex plane defined over $\mathbf{R}$ induces a morphism $\pi: Q_{3} \rightarrow \mathbf{P}_{\mathbf{C}}^{2}$, defined over $\mathbf{R}$, which is an isomorphism except two complex lines, defined over $\mathbf{R}$, of $\hat{Q}_{3}$ get contracted to two points. $\varepsilon$ and $\pi$ induce maps $\bar{\varepsilon}: \hat{Q}_{3} / \tau \rightarrow Q_{3} / \tau$ and $\bar{\pi}: \hat{Q}_{3} / \tau \rightarrow \mathbf{P}_{\mathbf{C}}^{2} / \tau$ which are diffeomorphisms except one or two submanifolds of $\hat{Q}_{3} / \tau$, each diffeomorphic to a closed 2-ball, are contracted to points. This implies $S^{4} \approx$ $Q_{3} / \tau \approx \hat{Q}_{3} / \tau \approx \mathbf{P}_{\mathbf{C}}^{2} / \tau$.

4. Finally there are 5 connected families of nonsingular cubics in $\mathbf{P}_{\mathbf{C}}^{3}$ defined over $\mathbf{R}$ ([5]). If we blow up in $\mathbf{P}_{\mathbf{C}}^{2} 6$ real points (in general position), or 4 real points and 2 conjugate nonreal points, or 2 real points and 4 conjugate nonreal points, or 6 conjugate nonreal points, we obtain 4 surfaces defined over $\mathbf{R}$ which we shall call, in order, $C_{1}, C_{2}, C_{3}, C_{4}$. There is an isomorphism defined over $\mathbf{R}$ of $C_{i}$ with a nonsingular cubic of $\mathbf{P}_{\mathbf{C}}^{3}$, defined over $\mathbf{R}$, which we shall also denote $C_{i}$. This isomorphism can be constructed as follows: consider the linear space of all the cubic curves in $\mathbf{P}_{\mathbf{C}}^{2}$ passing through the six points in question. Since the configuration of the six points is invariant under conjugation if a cubic passes through them so will the conjugate: hence that linear space will have a basis composed of cubics defined over $\mathbf{R}$. If $G_{l}\left(x_{0}, x_{1}, x_{2}\right), i=0,1,2,3$, is such a basis we consider the map

$$
\left(x_{0}: x_{1}: x_{2}\right) \rightarrow\left(G_{0}\left(x_{0}, x_{1}, x_{2}\right): G_{1}\left(x_{0}, x_{1}, x_{2}\right): \cdots: G_{3}\left(x_{0}, x_{1}, x_{2}\right)\right) \text {. }
$$

This map is, as easily seen, well defined on the complement of the set of the six points and is therefore an open immersion; the closure of its image will be a surface defined over $\mathbf{R}$, since $\phi$ commutes with conjugation, and will be in fact our surface $C_{i}$. Let $\hat{\mathbf{P}}_{\mathbf{C}}^{2}$ be the variety obtained from $\mathbf{P}_{\mathbf{C}}^{2}$ by blowing up the six points: conjugation of $\mathbf{P}_{\mathbf{C}}^{2}$ will extend to conjugation of $\hat{\mathbf{P}}_{\mathbf{C}}^{2}$ and $\phi$ will extend to an isomorphism of $\hat{\mathbf{P}}_{\mathbf{C}}^{2}$ with $C_{i}$ which commutes with conjugation. So $C_{i \mathbf{R}}$ will be diffeomorphic to $\hat{\mathbf{P}}_{\mathbf{R}}^{2}$ : this manifold is obtained from $\mathbf{P}_{\mathbf{R}}^{2}$ by replacing a disk around each of the real points which are blown up with two copies of $\mathbf{R}^{2}$ glued together by $x^{\prime}=x y, y^{\prime}=1 / x$, i.e. with a Möbius strip. Hence we have that $C_{i \mathbf{R}}$ is a connected compact nonorientable real surface which has $\operatorname{dim}_{\mathbf{R}} H^{1}\left(C_{i \mathbf{R}}, \mathbf{R}\right)=8-2 i(i=$ $1,2,3,4)$. We shall call $F_{i}$ the family to which $C_{i}$ belongs.

Let $C_{5}$ be defined by

$$
2 x^{3}+y^{3}+z^{3}-x^{2}+y^{2}+z^{2}+\lambda=0
$$

where $\lambda$ is any real number s.t. $0<\lambda<1 / 27$. 
If we put $x^{\prime}=x+\frac{1}{3}, y^{\prime}=y, z^{\prime}=z,(* * *)$ becomes:

$$
2 x^{\prime 3}+y^{\prime 3}+z^{\prime 3}+x^{\prime 2}+y^{\prime 2}+z^{\prime 2}+\lambda-1 / 27=0
$$

and we know from $\$ 2$ that the real solution locus of this equation has an $S^{2}$ as component (for $0<\lambda<1 / 27$ ); on the other hand there is an $\varepsilon>0$ s.t. if $-\varepsilon<\lambda_{0}<0$ the cubic given by $2 x^{3}+y^{3}+z^{3}-\mu x^{2}+y^{2}+z^{2}+$ $\lambda_{0}=0$ is nonsingular for $0 \leq \mu \leq 1$. Now the real locus of $2 x^{3}+y^{3}+z^{3}$ $+y^{2}+z^{2}+\lambda_{0}=0$ is diffeomorphic to $\mathbf{P}_{\mathbf{R}}^{2}$ as seen by considering the projection $(x, y, z) \rightarrow(y, z)$. It follows that $C_{5 \mathbf{R}} \approx \mathbf{P}_{\mathbf{R}}^{2} \cup S^{2}$ (the union being disjoint) and $C_{5}$ belongs to the remaining family $F_{5}$. Also we see that $C_{5}$ can be connected to a $C_{4}$ through a family which satisfies the conditions of the second case envisaged in $\S 2$. If $C_{i}$ and $C_{j}$ belong to adjacent families we must have, as we have seen, $\left|\chi\left(C_{i \mathbf{R}}, \mathbf{R}\right)-\chi\left(C_{j \mathbf{R}}, \mathbf{R}\right)\right|=2$ so that $F_{i}$ can only be adjacent to $F_{i-1}$ or $F_{i+1}$. Moreover since $C_{i \mathbf{R}}$ is connected for $i \leq 4$ when we connect through a family as in $\$ 2$ an element of $F_{i-1}$ with an element of $F_{i}$ for $i \leq 4$ we must always be in the second case. As in the above proof of Kuiper's theorem we can get at once $C_{1} / \tau \approx S^{4}$; from this and from what we just remarked it follows that $C_{i} / \tau \approx$ connected sum of $i-1$ copies of $\overline{\mathbf{P}}_{\mathbf{C}}^{2}$ for $i=2,3,4,5$.

(One can also proceed directly if $i \leq 4$ since one has maps $\bar{\varepsilon}_{i}$ : $C_{i} / \tau \rightarrow \mathbf{P}_{\mathbf{C}}^{2} / \tau \approx S^{4}$ which are diffeomorphisms except that $8-2 i$ closed 2-disks and $i-1 \overline{\mathbf{P}}_{\mathbf{C}}^{2}$ get contracted to points.)

As a final remark we mention that a cubic surface

$$
x^{3}+y^{3}+z^{3}+x^{2}+y^{2}+z^{2}+\lambda=0
$$

belongs to the family $F_{4}$ for $\lambda>0$ and to the family $F_{5}$ for $-4 / 27<\lambda<0$. So $C_{5} / \tau$ can also be seen as obtained from $C_{4} / \tau$ by removing a degree 1 disk bundle over $\mathbf{P}_{\mathbf{R}}^{2}$ and replacing it by a degree 4 complex line bundle over $\mathbf{P}_{\mathbf{C}}^{1}$.

We wish to thank Professors H. Clemens and R. Stern for their most helpful advice.

\section{REFERENCES}

[1] G. E. Bredon, Introduction to Compact Transformation Groups, Academic Press, 1972.

[2] D. A. Gudkov, The topology of real projective algebraic varieties, Russian Math. Surveys, 29, 4(1974), 1-79.

[3] N. Kuiper, The quotient space of $\mathbf{C P}(2)$ by complex conjugation is the 4-sphere, Math. Ann., 208 (1974), 175-177.

[4] J. Milnor, Singular points of complex hypersurfaces, Ann. Math. Studies, 61, Princeton, 1968. 
[5] O. A. Oleinik, Estimates of the Betti numbers of real algebraic hypersurfaces, Mat. Sbornik N.S., 28(70) (1951), 635-640.

[6] B. Segre, The Non Singular Cubic Surfaces, Oxford 1942.

[7] R. Thom, Sur l'homologie des variétés algébriques réelles, Differential and Combinatorial Topology (Morse Symposium, S. Cairns, ed.) Princeton Univ. Press, 1965, pp. 255-265.

Received March 29, 1982 and in revised form May 20, 1982. Research partially supported by the Consiglio Nazionale delle Ricerche, Italia.

\section{UNIVERSITA DI TRENTO}

Povo (TN) ITALIA 


\title{
PACIFIC JOURNAL OF MATHEMATICS EDITORS
}

\author{
DONALD BABBITT (Managing Editor) \\ University of California \\ Los Angeles, CA 90024 \\ Hugo Rossi \\ University of Utah \\ Salt Lake City, UT 84112 \\ C. C. MOORE and Arthur Ogus \\ University of California \\ Berkeley, CA 94720
}

\author{
J. Dugundir \\ Department of Mathematics \\ University of Southern California \\ Los Angeles, CA 90089-1113
}

R. FINN and H. SAMELSON

Stanford University

Stanford, CA 94305

ASSOCIATE EDITORS
R. ARENS
E. F. BECKENBACH
B. H. NeumanN
F. WOLF
K. YosHIDA (1906-1982)

\section{SUPPORTING INSTITUTIONS}

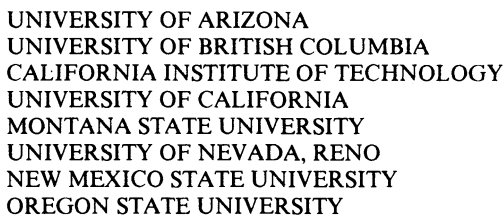

\author{
UNIVERSITY OF OREGON \\ UNIVERSITY OF SOUTHERN CALIFORNIA \\ STANFORD UNIVERSITY \\ UNIVERSITY OF HAWAII \\ UNIVERSITY OF TOKYO \\ UNIVERSITY OF UTAH \\ WASHINGTON STATE UNIVERSITY \\ UNIVERSITY OF WASHINGTON
}

The Supporting Institutions listed above contribute to the cost of publication of this Journal, but they are not owners or publishers and have no responsibility for its content or policies.

Mathematical papers intended for publication in the Pacific Journal of Mathematics should be in typed form or offset-reproduced (not dittoed), double spaced with large margins. Please do not use built up fractions in the text of the manuscript. However, you may use them in the displayed equations. Underline Greek letters in red, German in green, and script in blue. The first paragraph must be capable of being used separately as a synopsis of the entire paper. In particular it should contain no bibliographic references. Please propose a heading for the odd numbered pages of less than 35 characters. Manuscripts, in triplicate, may be sent to any one of the editors. Please classify according to the scheme of Math. Reviews, Index to Vol. 39. Supply name and address of author to whom proofs should be sent. All other communications should be addressed to the managing editor, or Elaine Barth, University of California, Los Angeles, California 90024.

There are page-charges associated with articles appearing in the Pacific Journal of Mathematics. These charges are expected to be paid by the author's University, Government Agency or Company. If the author or authors do not have access to such Institutional support these charges are waived. Single authors will receive 50 free reprints; joint authors will receive a total of 100 free reprints. Additional copies may be obtained at cost in multiples of 50 .

The Pacific Journal of Mathematics is issued monthly as of January 1966. Regular subscription rate: \$132.00 a year (6 Vol., 12 issues). Special rate: $\$ 66.00$ a year to individual members of supporting institutions.

Subscriptions, orders for numbers issued in the last three calendar years, and changes of address should be sent to Pacific Journal of Mathematics, P.O. Box 969, Carmel Valley, CA 93924, U.S.A. Old back numbers obtainable from Kraus Periodicals Co., Route 100, Millwood, NY 10546.

The Pacific Journal of Mathematics ISSN 0030-8730 is published monthly by the Pacific Journal of Mathematics at P.O. Box 969, Carmel Valley, CA 93924. Application to mail at Second-class postage rates is pending at Carmel Valley, California, and additional mailing offices. Postmaster: Send address changes to Pacific Journal of Mathematics, P. O. Box 969, Carmel Valley, CA 93924.

PUBLISHED BY PACIFIC JOURNAL OF MATHEMATICS, A NON-PROFIT CORPORATION

Copyright $(1984$ by Pacific Journal of Mathematics 


\section{Pacific Journal of Mathematics}

Vol. 110, No. $2 \quad$ October, 1984

Robert A. Bekes, The range of convolution operators $\ldots \ldots \ldots \ldots \ldots 257$

Dennis K. Burke and Sheldon Davis, Subsets of ${ }^{\omega} \omega$ and generalized metric

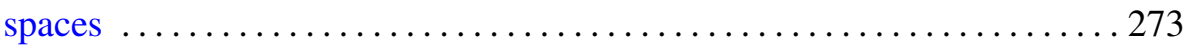

Giovanni Emmanuele, A remark on a paper: "Common fixed points of nonexpansive mappings by iteration" $\ldots \ldots \ldots \ldots \ldots \ldots \ldots \ldots \ldots 283$

I. Erdélyi and Sheng-Wang Wang, On strongly decomposable operators . . . 287

Gerhard Gierz, Injective Banach lattices with strong order units . . . . . . . 297

Maurizio Letizia, Quotients by complex conjugation of nonsingular quadrics and cubics in $\mathbf{P}_{\mathbf{C}}^{3}$ defined over $\mathbf{R} \ldots \ldots \ldots \ldots \ldots \ldots \ldots \ldots \ldots \ldots$

P. H. Maserick and Franciszek Hugon Szafraniec, Equivalent definitions

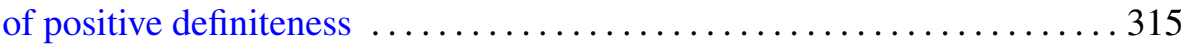

Costel Peligrad and S. Rubinstein, Maximal subalgebras of $C^{*}$-crossed

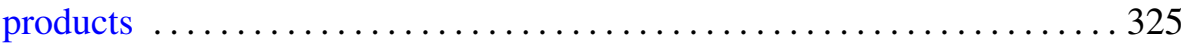

Derek W. Robinson and Sadayuki Yamamuro, Hereditary cones, order

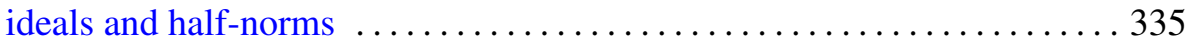

Derek W. Robinson and Sadayuki Yamamuro, The Jordan decomposition

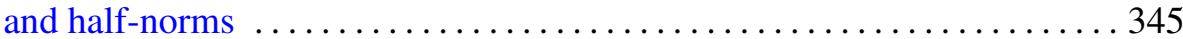

Richard Rochberg, Interpolation of Banach spaces and negatively curved

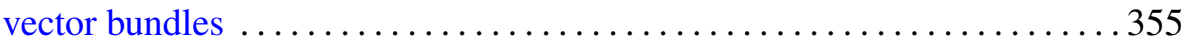

Dale Rolfsen, Rational surgery calculus: extension of Kirby's theorem 377

Walter Iaan Seaman, Helicoids of constant mean curvature and their Gauss maps

Diana Shelstad, Endoscopic groups and base change $\mathbf{C} / \mathbf{R}$

Jerrold Norman Siegel and Frank Williams, Numerical invariants of homotopies into spheres

Alladi Sitaram, Some remarks on measures on noncompact semisimple Lie groups

Teruhiko Soma, Atoroidal, irreducible 3-manifolds and 3-fold branched coverings of $S^{3}$

Jan de Vries, On the $G$-compactification of products

Hans Weber, Topological Boolean rings. Decomposition of finitely additive set functions 